\title{
Comparison of Monoexponential, Biexponential, Stretched-Exponential, and Kurtosis Models of Diffusion- Weighted Imaging in Differentiation of Renal Solid Masses
}

\author{
Jianjian Zhang, MD*, Shiteng Suo, MS*, Guiqin Liu, MD, Shan Zhang, MD, Zizhou Zhao, BS, \\ Jianrong $\mathrm{Xu}, \mathrm{MD}, \mathrm{PhD}$, Guangyu Wu, MD \\ All authors: Department of Radiology, Renji Hospital, School of Medicine, Shanghai Jiaotong University, Shanghai, China
}

Objective: To compare various models of diffusion-weighted imaging including monoexponential apparent diffusion coefficient $(A D C)$, biexponential (fast diffusion coefficient $\left[D_{f}\right]$, slow diffusion coefficient $\left[D_{s}\right]$, and fraction of fast diffusion), stretched-exponential (distributed diffusion coefficient and anomalous exponent term $[\alpha]$ ), and kurtosis (mean diffusivity and mean kurtosis $[M K]$ ) models in the differentiation of renal solid masses.

Materials and Methods: A total of 81 patients (56 men and 25 women; mean age, 57 years; age range, 30-69 years) with 18 benign and 63 malignant lesions were imaged using $3 T$ diffusion-weighted MRI. Diffusion model selection was investigated in each lesion using the Akaike information criteria. Mann-Whitney $U$ test and receiver operating characteristic (ROC) analysis were used for statistical evaluations.

Results: Goodness-of-fit analysis showed that the stretched-exponential model had the highest voxel percentages in benign and malignant lesions ( $90.7 \%$ and $51.4 \%$, respectively). ADC, $D_{s}$, and MK showed significant differences between benign and malignant lesions $(p<0.05)$ and between low- and high-grade clear cell renal cell carcinoma (ccRCC) $(p<0.05)$. $\alpha$ was significantly lower in the benign group than in the malignant group $(p<0.05)$. All diffusion measures showed significant differences between $\operatorname{ccRCC}$ and non-ccRCC $(p<0.05)$ except $D_{f}$ and $\alpha(p=0.143$ and 0.112 , respectively). $\alpha$ showed the highest diagnostic accuracy in differentiating benign and malignant lesions with an area under the ROC curve of 0.923 , but none of the parameters from these advanced models revealed significantly better performance over ADC in discriminating subtypes or grades of renal cell carcinoma (RCC) $(p>0.05)$.

Conclusion: Compared with conventional diffusion parameters, $\alpha$ may provide additional information for differentiating benign and malignant renal masses, while ADC remains the most valuable parameter for differentiation of RCC subtypes and for cCRCC grading.

Keywords: Magnetic resonance imaging; Diffusion-weighted imaging; Differentiation; Renal cell carcinoma; Renal masses

\section{INTRODUCTION}

Solid renal masses are the most common neoplasms of the urinary system, with renal cell carcinoma (RCC) accounting for nearly $90 \%$ of malignancies. Additionally, angiomyolipoma (AML) comprises $18-59 \%$ of resected benign solid tumors $(1,2)$. Distinguishing benign and malignant renal lesions, different tumor types, and different grades has clinical significance in determining the appropriate treatment strategy and evaluating prognosis

Received July 25, 2018; accepted after revision January 9, 2019.

This study was supported by the National Natural Science Foundation of China (81601487).

*These authors contributed equally to this work.

Corresponding author: Guangyu Wu, MD, Department of Radiology, Renji Hospital, School of Medicine, Shanghai Jiaotong University, 160 Pujian Road, Shanghai 200127, China.

- Tel: (8621) 68383864 • Fax: (8621) 68383864•E-mail: danielrau@163.com

This is an Open Access article distributed under the terms of the Creative Commons Attribution Non-Commercial License (https:// creativecommons.org/licenses/by-nc/4.0) which permits unrestricted non-commercial use, distribution, and reproduction in any medium, provided the original work is properly cited. 
$(3,4)$. Conventional computed tomography (CT) and magnetic resonance imaging (MRI) are routinely used in the evaluation of renal lesions. However, CT and MRI sometimes fail to provide reliable prediction when lesions lack typical imaging features. Moreover, approximately $16-33 \%$ of nephrectomies are performed on benign lesions (2).

Diffusion-weighted imaging (DWI) is a powerful technique for exploring biological microstructures. The apparent diffusion coefficient (ADC), obtained from DWI with the monoexponential model, has been widely used to characterize renal lesions (5-7). However, several studies have reported contradictory results using ADC to differentiate benign and malignant lesions $(8,9)$. Previous studies have suggested that advanced fitting models, such as biexponential, stretched-exponential, or kurtosis models might provide more accurate information regarding water diffusion $(10,11)$. The biexponential model, described by Le Bihan et al. (12), might allow separation of fast and slow diffusion components, which reflect capillary perfusion and tissue diffusion, respectively. The metrics derived from the biexponential model might be superior to $A D C$ in renal tumor diagnosis, pathological subtyping, and grade prediction $(13,14)$. Furthermore, Bennett et al. (15) proposed the stretched-exponential model, which was used to evaluate distributed diffusion and intravoxel heterogeneity. As one of the most popular non-Gaussian models, the diffusion kurtosis model has been used to reflect the complexity of tissue microstructure and provide a more comprehensive characterization of water diffusion in multiple organs such as the brain, prostate, and breast (16-18). These advanced models can fit the signal curve more precisely, reflecting tissue characteristics in greater detail. Although several studies on the role of DWI models in characterizing renal tumors have been done, they have generally used one or two models such as the monoexponential and/or biexponential models $(19,20)$. Another recent research paper also investigated the stretched-exponential model but here the model was used simply to differentiate RCC and AML (21). In our study, we aimed to apply four signal attenuation models (monoexponential, biexponential, stretched-exponential, and kurtosis models) to DWI data from renal lesions to determine the optimal model fitting the multiple b-value signal decay curve and quantitatively compare the potential of diffusion parameters in differentiating benign and malignant renal lesions, RCC subtyping, and clear cell RCC (ccRCC) grading.

\section{MATERIALS AND METHODS}

\section{Patients}

This retrospective study was approved by our Institutional Review Board, and written informed consent was obtained from all patients. One hundred and sixteen patients with known or suspected renal lesions at ultrasonography (US), CT, or both US and CT were enrolled in the study from June 2014 to December 2015. The inclusion criteria were as follows: 1) patients had complete information and received no treatment before MRI and 2) surgery was performed within two weeks after MRI examination and a pathologic confirmation was made. The exclusion criteria were as follows: 1) poor-quality images with obvious artifacts (n $=8), 2$ ) anti-tumor therapy and biopsy performed before MRI $(n=5), 3)$ no dynamic contrast-enhanced (DCE) data available $(n=10), 4)$ solid components that were difficult to characterize due to their small size $\left(<20 \mathrm{~mm}^{2}\right)(\mathrm{n}=9)$, and 5) massive fat-containing masses that could easily have been diagnosed as AML $(n=3)$. Ultimately, a total of 81 patients (mean age, 57 years; range, 30-69 years), including 56 men (mean age, 55 years; range, 30-69 years) and 25 women (mean age, 64 years; range, 51-68 years), were included. Only one lesion was found in each included patient. Histopathologic results were obtained from pathology reports.

\section{MRI Examination}

Patients were instructed to fast for $4-5$ hours before the examination. All scans were performed in the supine position using a 3T magnetic resonance scanner (Ingenia; Philips Medical Systems, Best, the Netherlands). The following sequences were used: axial turbo spin-echo T1weighted imaging, axial and coronal turbo spin-echo T2weighted imaging, axial DWI, and axial DCE imaging. DWIs with fat suppression were collected using a singleshot spin-echo echo-planar imaging sequence as follows: repetition time/echo time, 2000/86 ms; matrix, $152 \times 137$; in-plane resolution, $2 \times 2 \mathrm{~mm}$; section thickness, $5 \mathrm{~mm}$; parallel acquisition with acceleration factor, 2; b-values, $0,30,50,100,250,500,1000$, and $2000 \mathrm{sec} / \mathrm{mm}^{2}$; and acquisition time, 8 minutes 30 seconds. Sensitizing diffusion gradients were applied in the three orthogonal directions, and trace DWIs were generated. DCE imaging acquisitions were performed before and at three consecutive points after injection of gadolinium chelate (gadopentetate dimeglumine, Magnevist ${ }^{\circledR}$; Bayer Healthcare, Berlin, 
Germany) at a dose of $0.1 \mathrm{mmol} / \mathrm{kg}$ at a rate of $2 \mathrm{~mL} / \mathrm{sec}$.

\section{Image Analysis}

DWI data were transferred to a personal computer and processed with software (MatLab; MathWorks, Natick, MA, USA). The corresponding mathematical expressions are shown as follows:

1) Monoexponential model:

$$
S(b)=S_{0} \exp (-b A D C)
$$

where $S(b)$ and $S_{0}$ denote signal intensities with and without diffusion weighting, respectively, and $b$ is the diffusion-sensitizing factor.

2) Biexponential model:

$$
S(b)=S_{0}\left(f \exp \left[-b D_{f}\right]+[1-f] \exp \left[-b D_{s}\right]\right)
$$

where $D_{f}$ and $D_{s}$ represent fast diffusion coefficient and slow diffusion coefficient, respectively. $f$ represents the fraction of fast diffusion $(0 \leq f \leq 1)$.

3) Stretched-exponential model:

$$
\mathrm{S}(\mathrm{b})=\mathrm{S}_{0} \exp \left(-[\mathrm{bDDC}]^{\alpha}\right)
$$

where DDC represents distributed diffusion coefficient, and $\alpha$ represents the anomalous exponent term $(0 \leq \alpha \leq 1)$.

4) Diffusion kurtosis model:

$$
S(b)=S_{0} \exp \left(-b M D+b^{2} M D^{2} M K / 6\right)
$$

where MD represents mean diffusivity, and MK represents mean kurtosis.

Images were independently analyzed by two renal radiologists (with 5 and 7 years of experience, respectively) who were blinded to patients' pathologic findings. For each lesion, three regions of interest (ROIs) (the slice with maximal tumor dimension and its upper and lower slices) were placed in different enhancing solid components on DWI $b=0$ images, with reference to DCE to exclude areas of necrosis, cysts, hemorrhage and calcifications. The median size ROI was $65.7 \mathrm{~mm}^{2}$ with range of $34.4-308.6 \mathrm{~mm}^{2}$ (benign; median size $54.3 \mathrm{~mm}^{2}$, size range $34.4-210.1 \mathrm{~mm}^{2}$ : malignant; median size $81.6 \mathrm{~mm}^{2}$, size range 45.4-308.6 $\mathrm{mm}^{2} ; p=0.14$ using Mann-Whitney U-test). The median value of each parameter from all pixels within the ROI was used for statistical analysis to reduce the sensitivity to outlier values.

\section{Statistical Analysis}

Statistical analyses were performed with MedCalc v. 12.7 (MedCalc Software, Mariakerke, Belgium). The goodnessof-fit of the four models was compared using the Akaike information criterion (22). For each voxel within the ROI, the best fitting curve was determined through calculating its vertical distances to the four curves (the shortest distance means the best fitting). The highest percentage of overall number of voxels indicated the optimal model. The interobserver variability for parameter measurements was assessed by using the intraclass correlation coefficient (ICC: 0.00-0.20, poor agreement; $0.21-0.40$, fair; $0.41-0.60$, moderate; 0.61-0.80, good; and 0.81-1.00, excellent) (23). A non-normal distribution was confirmed for all parameters except ADC, $\alpha$, and $D_{s}$ according to the Shapiro-Wilk test; nonparametric statistical tests were used for further analysis. Mann-Whitney U-tests were used to compare the diffusion parameters of patient subgroups assigned in terms of tumor types (benign and malignant), RCC subtypes (ccRCC and non-ccRCC), and $c C R C C$ grading (low- and high-grade). Receiver operating characteristic (ROC) curves were created, and areas under the ROC curve (AUCS) were compared between $A D C$ and other diffusion parameters by using the method developed by DeLong et al. (24). The maximum Youden index was used to determine the optimal sensitivity and specificity, as well as the corresponding cut-off value. A $p$ value $<0.05$ was considered significant.

\section{RESULTS}

\section{Histopathologic Results}

Of the 81 renal lesions, 18 lesions (22.2\%) were categorized as benign and 63 lesions $(77.8 \%)$ were categorized as malignant. The benign group comprised 15 AMLs (83.3\%), two oncocytomas (11.1\%), and one hyperplasia of fiber tissue (5.6\%). Of the 63 malignant lesions, 46 ccRCCs $(73.0 \%)$, 11 papillary renal cell carcinoma (pRCCs) (17.5\%), and 6 chromophobe renal cell carcinoma (chRCCs) (9.5\%) were identified. Patients with ccRCC were assigned to two groups according to the Fuhrman nuclear grading system: low-grade (25 in grade I and 14 in grade II) and high-grade ( 3 in grade III and 4 in grade IV). A pathologic examination confirmed type I pRCC in 5 patients ( 2 in grade I and 3 in grade II) and type II pRCC in 6 patients ( 2 in grade I and 4 in grade II). The 
average diameter of the 81 lesions was $4.1 \mathrm{~cm}$, with a range of 1.2-12.7 cm (benign: median size $3.4 \mathrm{~cm}$, range 1.2-6.4 $\mathrm{cm}$; malignant: median size $4.9 \mathrm{~cm}$, range $2.6-12.7 \mathrm{~cm}$ ).

\section{Goodness-of-Fit Assessment}

According to Table 1, the assessment of goodness-of-fit showed that the voxel percentages in benign and malignant lesions described by the stretched-exponential model were $90.7 \%$ and $51.4 \%$, respectively. Monoexponential and biexponential models demonstrated relatively poor performance in fitting the diffusion-weighted (DW) dataset, with voxel percentages of $0.6 \%$ and $0 \%$, respectively, for benign lesions and $2.0 \%$ and $0 \%$, respectively, for malignant lesions. The kurtosis model fitted the voxels better but also

Table 1. Voxel Percentages Preferred by Four Models in Renal Lesions

\begin{tabular}{lcccr}
\hline \multicolumn{1}{c}{ Model } & Monoexponential & Biexponential & Stretched-Exponential & Kurtosis \\
\hline Benign (\%) & $0.6(6.5)$ & $0(8.5)$ & $90.7(38.4)$ & $3.9(13.9)$ \\
Malignant (\%) & $2.0(11.0)$ & $0(14.1)$ & $51.4(24.4)$ & $30.3(32.8)$ \\
\hline
\end{tabular}

Data are medians with interquartile range in parentheses.
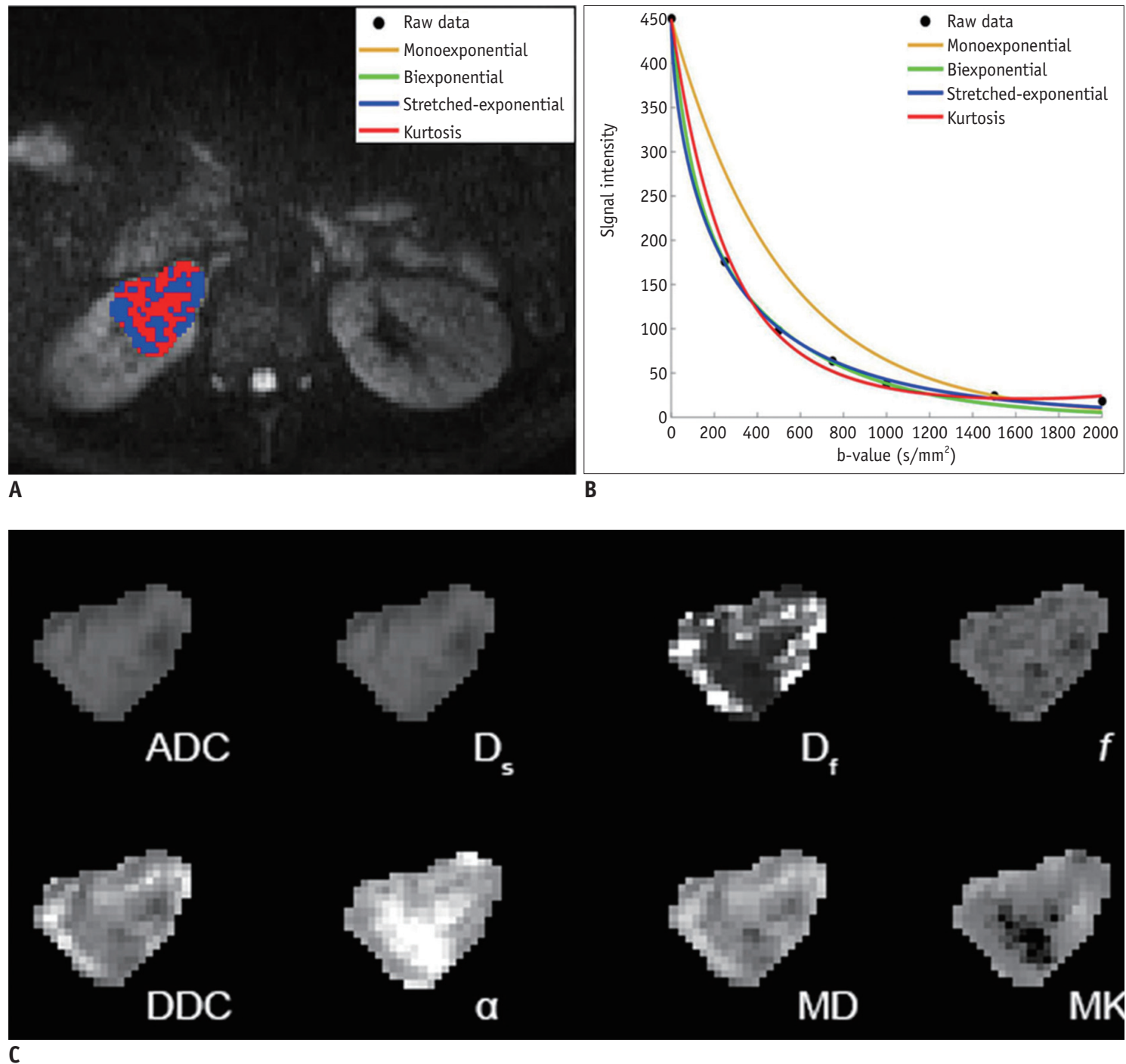

Fig. 1. Clear cell renal cell carcinoma (grade II) in right kidney in 39-year-old man.

A. Voxels preferred by monoexponential, biexponential, stretched-exponential, and kurtosis models in lesion. B. Plot of decay of diffusionweighted signal intensity as function of b-value from representative voxel within ROI. C. Multiparametric diffusion parameter maps within ROI. $A D C=$ apparent diffusion coefficient, $D D C=$ distributed diffusion coefficient, $D_{f}=$ fast diffusion coefficient, $D s=$ slow diffusion coefficient, $f=$ fraction of fast diffusion, $M D=$ mean diffusivity, $M K=$ mean kurtosis, $R O I=$ region of interest, $\alpha=$ anomalous exponent term 

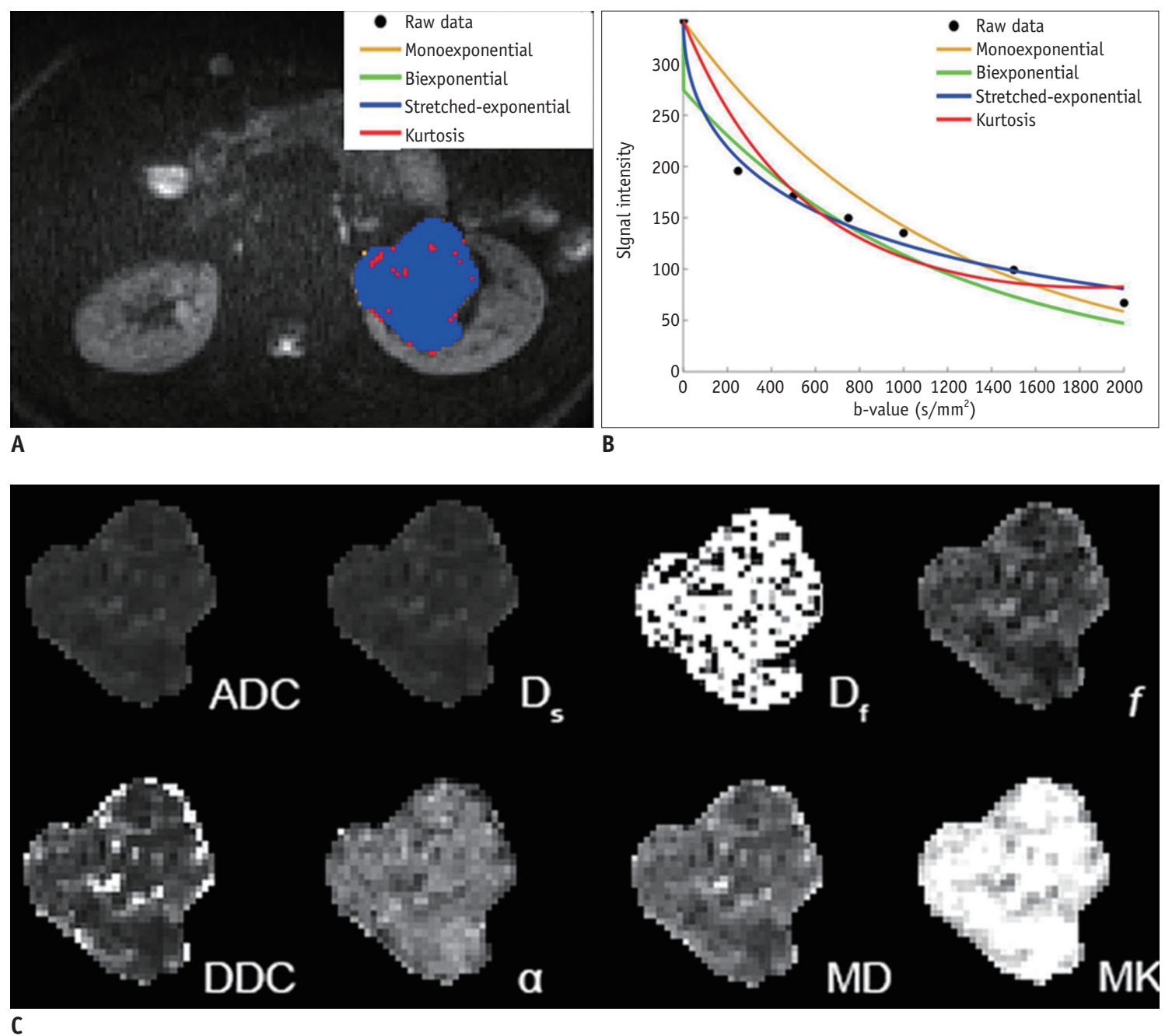

Fig. 2. Angiomyolipoma in left kidney in 55-year-old woman.

A. Voxels preferred by monoexponential, biexponential, stretched-exponential and kurtosis models in lesion. B. Plot of decay of diffusionweighted signal intensity as function of b-value from representative voxel within ROI. C. Multiparametric diffusion parameter maps within ROI.

had low voxel percentages of $3.9 \%$ and $30.3 \%$ for benign and malignant lesions, respectively. The fitting curves of different models and parameter maps for ccRCC and AML are displayed in Figures 1 and 2.

\section{Interobserver Agreement}

All parameter measurements resulted in good to excellent interobserver agreement. Excellent interobserver agreement was found for $A D C, D_{5}, D D C, \alpha, M D$, and $M K(I C C=0.881$, $0.890,0.904,0.869,0.898$, and 0.852 , respectively). Good interobserver agreement was found for $D_{f}$ and $f(I C C=0.712$ and 0.795 , respectively).

\section{Association between Diffusion Parameters and Pathologic Diagnoses}

Mann-Whitney U-tests revealed that $\mathrm{ADC}, \mathrm{D}_{\mathrm{s}}, \alpha$, and $\mathrm{MK}$ differed significantly between benign and malignant lesions (all Ps < 0.05). No significant difference was found between the two groups for $f_{,} D_{f}, D D C$, and MD $(p>0.05)$. As shown in Table 2, AUC values were 0.731 for ADC, 0.752 for $D_{s^{\prime}}$ 0.567 for $D_{f}, 0.496$ for $f, 0.543$ for DDC, 0.923 for $\alpha, 0.568$ for MD, and 0.803 for MK. $\alpha$ showed the highest diagnostic accuracy, the AUC value of which was significantly higher than that of $\operatorname{ADC}(p=0.001)$.

For comparisons of pathologic subtypes, all parameters showed significant differences between $\mathrm{ccRCC}$ and non$\operatorname{ccRCC}(p<0.05)$, except $D_{f}$ and $\alpha(p=0.143$ and 0.112 , respectively) (Table 3 ). The AUC values of $D_{s^{\prime}}, D D C, M D$, and $M K$ were $0.903,0.920,0.898$, and 0.851 , respectively, showing no significant differences compared with those of ADC (0.918) (Table 3). However, $D_{f}, f$, and $\alpha$, whose AUC values were 0.621 , 0.791 , and 0.631 , respectively, showed significantly lower 
Table 2. Diagnostic Performance of Diffusion Parameters in Differentiating Renal Lesions Types

\begin{tabular}{|c|c|c|c|c|c|c|c|c|}
\hline $\begin{array}{c}\text { Parameter } \\
\left(\times 10^{-3} \mathrm{~mm}^{2} / \mathrm{sec}\right)\end{array}$ & $A D C$ & $D_{s}$ & $D_{f}$ & $f^{*}$ & $\mathrm{DDC}$ & $\alpha^{*}$ & MD & $M K^{*}$ \\
\hline \multicolumn{9}{|l|}{ Benign vs. Malignant } \\
\hline \multirow{2}{*}{$\operatorname{AUC}(95 \% \mathrm{CI})$} & 0.731 & 0.752 & 0.567 & 0.496 & 0.543 & 0.923 & 0.568 & 0.803 \\
\hline & $(0.621-0.824)$ & $(0.644-0.841)$ & $(0.452-0.677)$ & $(0.383-0.609)$ & $(0.429-0.654)$ & $(0.842-0.971)$ & $(0.453-0.678)$ & $(0.700-0.883)$ \\
\hline$p^{\dagger}$ & Ref & 0.313 & 0.042 & 0.007 & $<0.001$ & 0.001 & $<0.001$ & 0.217 \\
\hline Sensitivity (\%) & 60.3 & 58.7 & 98.4 & 39.7 & 68.3 & 96.8 & 60.3 & 63.5 \\
\hline Specificity (\%) & 88.9 & 88.9 & 27.8 & 77.8 & 61.1 & 72.2 & 61.1 & 88.9 \\
\hline Cut-off value & 0.0014 & 0.0014 & 0.0416 & 0.3000 & 0.0019 & 0.5200 & 0.0025 & 0.7200 \\
\hline \multicolumn{9}{|l|}{ ccRCC vs. Non-ccRCC } \\
\hline \multirow{2}{*}{$\operatorname{AUC}(95 \% \mathrm{CI})$} & 0.918 & 0.903 & 0.621 & 0.791 & 0.920 & 0.631 & 0.898 & 0.851 \\
\hline & $(0.820-0.972)$ & $(0.802-0.963)$ & $(0.490-0.740)$ & $(0.670-0.883)$ & $(0.824-0.973)$ & $(0.500-0.749)$ & $(0.796-0.960)$ & (0.739-0.928) \\
\hline$p^{\dagger}$ & Ref & 0.237 & $<0.001$ & 0.014 & 0.865 & 0.001 & 0.641 & 0.117 \\
\hline Sensitivity (\%) & 80.4 & 80.4 & 73.9 & 76.7 & 84.8 & 52.2 & 95.7 & 89.1 \\
\hline Specificity (\%) & 94.1 & 94.1 & 52.9 & 70.6 & 94.1 & 82.4 & 76.5 & 76.5 \\
\hline Cut-off value & 0.0014 & 0.0014 & 0.0078 & 0.1800 & 0.0020 & 0.6600 & 0.0018 & 0.8000 \\
\hline \multicolumn{9}{|c|}{ Low-grade vs. high-grade } \\
\hline \multirow{2}{*}{ AUC $(95 \%$ CI $)$} & 0.780 & 0.802 & 0.630 & 0.663 & 0.707 & 0.712 & 0.568 & 0.823 \\
\hline & $(0.634-0.889)$ & $(0.658-0.905)$ & $(0.475-0.768)$ & $(0.509-0.796)$ & $(0.554-0.832)$ & $(0.560-0.836)$ & $(0.412-0.715)$ & $(0.680-0.920)$ \\
\hline$p^{\dagger}$ & Ref & 0.297 & 0.397 & 0.443 & 0.592 & 0.509 & 0.047 & 0.456 \\
\hline Sensitivity (\%) & 84.6 & 89.7 & 35.9 & 64.1 & 89.7 & 43.6 & 94.9 & 71.8 \\
\hline Specificity (\%) & 71.4 & 71.4 & 100 & 71.4 & 57.1 & 100 & 42.9 & 100 \\
\hline Cut-off value & 0.0014 & 0.0013 & 0.0083 & 0.2300 & 0.0021 & 0.6800 & 0.0019 & 0.6500 \\
\hline
\end{tabular}

Data are medians with interquartile range in parentheses. ${ }^{*} \mathrm{f}, \alpha$, and $\mathrm{MK}$ have no units, ${ }^{\dagger} p$ value from statistical comparison between AUC values of ADC (Ref) and other diffusion parameters by using method of DeLong et al. (24). AUC = area under receiver operating characteristic curve, $\mathrm{CI}=$ confidence intervals, $\mathrm{Ref}=$ reference

Table 3. Association between Diffusion Parameters and Pathologic Diagnoses

\begin{tabular}{|c|c|c|c|c|c|c|c|c|}
\hline $\begin{array}{c}\text { Parameter } \\
\left(\times 10^{-3} \mathrm{~mm}^{2} / \mathrm{sec}\right)\end{array}$ & $A D C$ & $D_{s}$ & $D_{f}$ & $f^{*}$ & DDC & $\alpha^{*}$ & MD & $M K^{*}$ \\
\hline Benign $(n=18)$ & $\begin{array}{c}0.0010 \\
(0.0004)\end{array}$ & $\begin{array}{c}0.0010 \\
(0.0004)\end{array}$ & $\begin{array}{c}0.0138 \\
(0.0414)\end{array}$ & $\begin{array}{c}0.2300 \\
(0.1400)\end{array}$ & $\begin{array}{c}0.0016 \\
(0.0023)\end{array}$ & $\begin{array}{c}0.4450 \\
(0.1600)\end{array}$ & $\begin{array}{c}0.0022 \\
(0.0013)\end{array}$ & $\begin{array}{c}0.9750 \\
(0.2900)\end{array}$ \\
\hline Malignant $(n=63)$ & $\begin{array}{c}0.0015 \\
(0.0006)\end{array}$ & $\begin{array}{c}0.0015 \\
(0.0006)\end{array}$ & $\begin{array}{c}0.0087 \\
(0.0135)\end{array}$ & $\begin{array}{c}0.2300 \\
(0.1900)\end{array}$ & $\begin{array}{c}0.0023 \\
(0.0014)\end{array}$ & $\begin{array}{c}0.6800 \\
(0.1300)\end{array}$ & $\begin{array}{c}0.0027 \\
(0.0012)\end{array}$ & $\begin{array}{c}0.6660 \\
(0.3000)\end{array}$ \\
\hline$p^{\dagger}$ & 0.003 & 0.001 & 0.388 & 0.959 & 0.578 & $<0.001$ & 0.420 & $<0.001$ \\
\hline $\operatorname{ccRCC}(n=46)$ & $\begin{array}{c}0.0016 \\
(0.0004)\end{array}$ & $\begin{array}{c}0.0016 \\
(0.0004)\end{array}$ & $\begin{array}{c}0.0095 \\
(0.0172)\end{array}$ & $\begin{array}{c}0.3250 \\
(0.1800)\end{array}$ & $\begin{array}{c}0.0024 \\
(0.0005)\end{array}$ & $\begin{array}{c}0.6550 \\
(0.1400)\end{array}$ & $\begin{array}{c}0.0028 \\
(0.0006)\end{array}$ & $\begin{array}{c}0.6200 \\
(0.1800)\end{array}$ \\
\hline Non-ccRCC $(n=17)$ & $\begin{array}{c}0.0010 \\
(0.0004)\end{array}$ & $\begin{array}{c}0.0010 \\
(0.0005)\end{array}$ & $\begin{array}{c}0.0078 \\
(0.0124)\end{array}$ & $\begin{array}{c}0.1500 \\
(0.1300)\end{array}$ & $\begin{array}{c}0.0012 \\
(0.0005)\end{array}$ & $\begin{array}{c}0.7000 \\
(0.0700)\end{array}$ & $\begin{array}{c}0.0016 \\
(0.0007)\end{array}$ & $\begin{array}{c}0.9000 \\
(0.1600)\end{array}$ \\
\hline$p^{\dagger}$ & $<0.001$ & $<0.001$ & 0.143 & $<0.001$ & $<0.001$ & 0.112 & $<0.001$ & $<0.001$ \\
\hline Low-grade $(n=39)$ & $\begin{array}{c}0.0017 \\
(0.0003)\end{array}$ & $\begin{array}{c}0.0017 \\
(0.0003)\end{array}$ & $\begin{array}{c}0.0087 \\
(0.0170)\end{array}$ & $\begin{array}{c}0.3300 \\
(0.1700)\end{array}$ & $\begin{array}{c}0.0025 \\
(0.0006)\end{array}$ & $\begin{array}{c}0.6700 \\
(0.1400)\end{array}$ & $\begin{array}{c}0.0028 \\
(0.0006)\end{array}$ & $\begin{array}{c}0.6000 \\
(0.1900)\end{array}$ \\
\hline High-grade $(n=7)$ & $\begin{array}{c}0.0014 \\
(0.0005)\end{array}$ & $\begin{array}{c}0.0011 \\
(0.0007)\end{array}$ & $\begin{array}{c}0.0116 \\
(0.0224)\end{array}$ & $\begin{array}{c}0.2200 \\
(0.1900)\end{array}$ & $\begin{array}{c}0.0021 \\
(0.0014)\end{array}$ & $\begin{array}{c}0.6000 \\
(0.1300)\end{array}$ & $\begin{array}{c}0.0028 \\
(0.0010)\end{array}$ & $\begin{array}{c}0.7500 \\
(0.1600)\end{array}$ \\
\hline$p^{\dagger}$ & 0.018 & 0.010 & 0.291 & 0.183 & 0.087 & 0.076 & 0.291 & 0.002 \\
\hline
\end{tabular}

Data are medians with interquartile range in parentheses. ${ }^{*} \mathrm{f}, \alpha$, and $\mathrm{MK}$ have no units, ${ }^{\dagger} p$ value from statistical comparison between benign and malignant lesions, between $c c R C C$ and non-ccRCC, between low-grade and high-grade ccRCC, respectively, by using MannWhitney $\mathrm{U}$ test. $A D C=$ apparent diffusion coefficient, $c c R C C=$ clear cell renal cell carcinoma, $D D C=$ distributed diffusion coefficient, $D_{f}$ $=$ fast diffusion coefficient, $D_{s}=$ slow diffusion coefficient, $f=$ fraction of fast diffusion, $M D=$ mean diffusivity, $M K=$ mean kurtosis, $\alpha=$ anomalous exponent term 
diagnostic accuracy than did $\operatorname{ADC}(p<0.05)$.

The results of comparative analysis of diffusion parameters between $\mathrm{ccRCC}$ with different grades were also displayed in Tables 2 and 3. $A D C, D_{s}$, and MK were significantly different between low-grade and high-grade groups (all Ps $<0.05$ ). Other parameters did not show significant differences between the two groups (all Ps >0.05). AUC values were 0.780 for $A D C, 0.802$ for $D_{s}, 0.630$ for $D_{f}, 0.663$ for $f, 0.707$ for DDC, 0.712 for $\alpha, 0.568$ for MD, and 0.823 for MK. The AUC values for $D_{s}$ and $M K$ were slightly higher than those for ADC, but there was no significant difference (all Ps > 0.05). Other parameters did not show significantly higher $A U C$ values than $A D C$.

\section{DISCUSSION}

In this study, the stretched-exponential model provided significantly better characterization of DW signal decay in both benign and malignant renal lesions than did the monoexponential, biexponential, or kurtosis model. This result was consistent with findings in other malignant tumors, including metastatic abdominal and pelvic tumors and endometrial cancer, indicating that significantly better fitting of the diffusion signals could be achieved by non-Gaussian models $(25,26)$. Although both the biexponential and kurtosis models obtained much poorer fit performances in relation in both benign and malignant lesions, it might be partly due to the tumor types, which did not have an extremely significant intravoxel incoherent motion or kurtosis effect, or the choice of the number and range of b-values used in the study. Moreover, model performance changes based on the number and definitions of its parameters. For instance, the microstructural content of tumor tissue is conventionally divided into three parts, including the perfusion-related lesion, extravascular extracellular space, and cellular compartment, and the latter two are classified as slow compartments (27). The biexponential model may not be sufficient to handle two slow compartments because it provides only one parameter for the evaluation of the slow diffusion component.

We showed that $\alpha$ had significantly greater diagnostic value in differentiating benign and malignant renal lesions than did conventional or other non-Gaussian distribution parameters. However, none of the parameters from these advanced models showed significantly superior performance over ADC in discriminating subtypes or grades of RCC. In particular, a showed a much lower AUC value than did ADC in differentiating $\mathrm{cCRCC}$ and non-ccRCC. As an anomalous exponent term, $\alpha$ is considered to provide a means of assessing inter-lesion heterogeneity. Therefore, this result is unsurprising because the tissue architecture, cell density, and microcirculation may show large variations between benign and malignant lesions, while the deviation between certain kinds of malignant tumors or different grades of ccRCC might be quite modest, leading to lower diagnostic values for those non-Gaussian diffusion parameters. Although the diagnostic performance in differential RCC subtypes is less satisfactory, fewer differences within malignant tumors could be an important factor for the superior performance in discrimination between benign and malignant tumors with the stretched-exponential model.

The monoexponential model is valuable in the evaluation of renal masses $(28,29)$. ADC is lower in malignant masses than in benign masses and this presumably indicates higher cellularity in malignancies (6). However, Ding et al. (20) reported that $\operatorname{CCRCC}$ had a significantly higher $A D C$ value than did non-cCRCC and AML, consistent with our results. cCRCC tumor cells are often interspersed with cystic and hemorrhagic areas and separated by interstitial spaces; thus, water molecules could diffuse relatively freely. In contrast, benign lesions and non-ccRCC usually present with compact tissue architecture and greater cellular density, leading to decreased $A D C$ values. As far as we know, $A D C$ values are mainly affected by both tumor cellularity and vascularity, but in tumor tissue, no final conclusion has been made on the precise proportion of each component to constitute $A D C$. Our current study also showed that the results of $A D C$ were consistent with those of $D_{s}$, which theoretically removes the influence of perfusion and may reflect the true diffusion coefficient. This finding suggests that the perfusion contribution to $A D C$ was small and that $A D C$ values can reflect the true diffusion coefficient to some extent, at least at the given $b$-values $\left(b=0-2000 \mathrm{sec} / \mathrm{mm}^{2}\right)$ in the present study.

$D_{f}$ and $f$, the other two important metrics in the biexponential model, are linked to perfusion and reflect the degree of tissue vascularity without the use of contrast agents. As for $D_{f}$, its diagnostic value has always been controversial $(13,14,30)$. In our study, we did not find a significant difference between any subject subgroups for $D_{f}$ values. Variations in the results might be related to the different sample sizes and the number and distribution of b-values in these studies. Additionally, artifacts from great vessels may also have an impact on this parameter. 
As such, further efforts are necessary to make this metric more reproducible and reliable. In contrast, the other perfusion-related parameter $f$ performed more reliably in recent studies, suggesting that it is associated with the enhancement degree of renal lesions, accurately separating enhancing cCRCC from hypoenhancing pRCC and cystic RCC (31). Similar to prior investigations, we found higher $f$ values in ccRCC than in non-cCRCC, indicating the hypervascularity of ccRCC.

Several studies using advanced diffusion models including the stretched-exponential and kurtosis models to probe the non-Gaussian water behavior in tissues, have been reported $(32,33)$. In our current study, lower $\alpha$ and higher MK values were observed in benign lesions than in malignant ones. Although Fujima et al. (34) also suggested an inverse relationship between $\alpha$ and MK, and that the two metrics might be similar in describing the pathologic characteristics of tumors, this conclusion was controversial. The performance of these two metrics in assessing the pathophysiologic state is not always consistent (35), which also occurred in our study. The diffusion kurtosis model uses a quadratic equation, whereas the stretchedexponential model uses the exponential function, and there is no definite deductive formula between the two parameters. Therefore, as mentioned in previous studies, the exact meanings of $\alpha$ and MK in vivo are not fully understood and were mainly referenced as metrics that could assess the tissue "complexity". Based on our results, we assume that $\alpha$ and MK may reflect information on a specific part of the "complexity". However, as the definition and extent of "complexity" remain unclear, the precise underlying biological mechanism needs to be further explored.

Our study has several limitations. One is the relatively small number of non-ccRCCs and high-grade ccRCCs. Additional studies with a large sample size are necessary to confirm the results. Another limitation is the lack of research on the value of contrast-enhanced MRI in this study. However, contrast-enhanced imaging might be inferior to DWI in renal tumor diagnosis, especially for complicated renal lesions, hemorrhagic lesions, or tumors showing overlap in the enhancement pattern $(28,36)$. Moreover, most patients with renal tumors are elderly individuals and may have renal dysfunction, which increases the risk of development of nephrogenic systemic fibrosis after contrast administration (37). Thus, DWI might be a reasonable alternative for these patients.

In conclusion, our results suggest that the stretched- exponential model provides the best fitting performance for DW-MRI data from both benign and malignant renal lesions. Compared with conventional diffusion parameters, $\alpha$ may provide additional information for differentiating benign and malignant renal masses, while ADC remains the most valuable parameter for the differentiation of $\mathrm{CCRCC}$ and non-ccRCC, as well as for ccRCC grading. However, at present, we have not identified a single DWI model that could be adequately powerful in characterizing renal masses. Therefore, combining these Gaussian diffusion and non-Gaussian diffusion parameters may be reasonable and helpful in improving therapeutic strategies and prognoses in clinical practice, however, these approaches need further research in the future.

\section{Conflicts of Interest}

The authors have no potential conflicts of interest to disclose.

\author{
ORCID iDs \\ Guangyu Wu \\ https://orcid.org/0000-0002-7043-2357 \\ Jianjian Zhang \\ https://orcid.org/0000-0003-3485-0495 \\ Shiteng Suo \\ https://orcid.org/0000-0003-2528-2836
}

\section{REFERENCES}

1. Sasiwimonphan K, Takahashi N, Leibovich BC, Carter RE, Atwell TD, Kawashima A. Small $(<4 \mathrm{~cm})$ renal mass: differentiation of angiomyolipoma without visible fat from renal cell carcinoma utilizing MR imaging. Radiology 2016;280:653

2. Kutikov A, Fossett LK, Ramchandani P, Tomaszewski JE, Siegelman ES, Banner MP, et al. Incidence of benign pathologic findings at partial nephrectomy for solitary renal mass presumed to be renal cell carcinoma on preoperative imaging. Urology 2006;68:737-740

3. Escudier B, Eisen T, Stadler WM, Szczylik C, Oudard S, Siebels $M$, et al. Sorafenib in advanced clear-cell renal-cell carcinoma. N Engl J Med 2007;356:125-134

4. Goyal A, Sharma R, Bhalla AS, Gamanagatti S, Seth A, Iyer VK, et al. Diffusion-weighted MRI in renal cell carcinoma: a surrogate marker for predicting nuclear grade and histological subtype. Acta Radiol 2012;53:349-358

5. Yu X, Lin M, Ouyang H, Zhou C, Zhang H. Application of ADC measurement in characterization of renal cell carcinomas with different pathological types and grades by $3.0 \mathrm{~T}$ diffusion- 
weighted MRI. Eur J Radiol 2012;81:3061-3066

6. Wang H, Cheng L, Zhang X, Wang D, Guo A, Gao Y, et al. Renal cell carcinoma: diffusion-weighted MR imaging for subtype differentiation at 3.0T. Radiology 2010;257:135-143

7. Zhang J, Tehrani YM, Wang L, Ishill NM, Schwartz LH, Hricak $H$. Renal masses: characterization with diffusion-weighted MR imaging--a preliminary experience. Radiology 2008;247:458464

8. Sevcenco S, Heinz-Peer G, Ponhold L, Javor D, Kuehhas FE, Klingler $\mathrm{HC}$, et al. Utility and limitations of 3-Tesla diffusionweighted magnetic resonance imaging for differentiation of renal tumors. Eur J Radiol 2014;83:909-913

9. Liu JH, Tian SF, Ju Y, Li Y, Chen AL, Chen LH, et al. Apparent diffusion coefficient measurement by diffusion weighted magnetic resonance imaging is a useful tool in differentiating renal tumors. BMC Cancer 2015;15:292

10. Bai Y, Lin Y, Tian J, Shi D, Cheng J, Haacke EM, et al. Grading of gliomas by using monoexponential, biexponential, and stretched exponential diffusion-weighted MR imaging and diffusion kurtosis MR imaging. Radiology 2016;278:496-504

11. Suo S, Cheng F, Cao M, Kang J, Wang M, Hua J, et al. Multiparametric diffusion-weighted imaging in breast lesions: association with pathologic diagnosis and prognostic factors. J Magn Reson Imaging 2017;46:740-750

12. Le Bihan D, Breton E, Lallemand D, Aubin ML, Vignaud J, Laval-Jeantet M. Separation of diffusion and perfusion in intravoxel incoherent motion MR imaging. Radiology 1988;168:497-505

13. Chandarana H, Lee VS, Hecht E, Taouli B, Sigmund EE. Comparison of biexponential and monoexponential model of diffusion weighted imaging in evaluation of renal lesions: preliminary experience. Invest Radiol 2011;46:285-291

14. Rheinheimer S, Stieltjes B, Schneider F, Simon D, Pahernik S, Kauczor $\mathrm{HU}$, et al. Investigation of renal lesions by diffusionweighted magnetic resonance imaging applying intravoxel incoherent motion-derived parameters--initial experience. Eur J Radiol 2012;81:e310-e316

15. Bennett KM, Schmainda KM, Bennett RT, Rowe DB, Lu H, Hyde JS. Characterization of continuously distributed cortical water diffusion rates with a stretched-exponential model. Magn Reson Med 2003;50:727-734

16. Raab P, Hattingen E, Franz K, Zanella FE, Lanfermann H. Cerebral gliomas: diffusional kurtosis imaging analysis of microstructural differences. Radiology 2010;254:876-881

17. Nogueira L, Brandão S, Matos E, Nunes RG, Loureiro J, Ramos I, et al. Application of the diffusion kurtosis model for the study of breast lesions. Eur Radiol 2014;24:1197-1203

18. Rosenkrantz AB, Sigmund EE, Johnson G, Babb JS, Mussi TC, Melamed J, et al. Prostate cancer: feasibility and preliminary experience of a diffusional kurtosis model for detection and assessment of aggressiveness of peripheral zone cancer. Radiology 2012;264:126-135

19. Parada Villavicencio C, Mc Carthy RJ, Miller FH. Can diffusionweighted magnetic resonance imaging of clear cell renal carcinoma predict low from high nuclear grade tumors. Abdom Radiol (NY) 2017;42:1241-1249

20. Ding $Y$, Zeng M, Rao S, Chen C, Fu C, Zhou J. Comparison of biexponential and monoexponential model of diffusionweighted imaging for distinguishing between common renal cell carcinoma and fat poor angiomyolipoma. Korean J Radiol 2016;17:853-863

21. Li H, Liang L, Li A, Hu Y, Hu D, Li Z, et al. Monoexponential, biexponential, and stretched exponential diffusion-weighted imaging models: quantitative biomarkers for differentiating renal clear cell carcinoma and minimal fat angiomyolipoma. $J$ Magn Reson Imaging 2017;46:240-247

22. Akaike H. [Data analysis by statistical models]. No To Hattatsu 1992;24:127-133

23. Ahlawat S, Khandheria P, Del GF, Morelli J, Subhawong TK, Demehri $S$, et al. Interobserver variability of selective regionof-interest measurement protocols for quantitative diffusion weighted imaging in soft tissue masses: comparison with whole tumor volume measurements. J Magn Reson Imaging 2016;43:446-454

24. DeLong ER, DeLong DM, Clarke-Pearson DL. Comparing the areas under two or more correlated receiver operating characteristic curves: a nonparametric approach. Biometrics 1988;44:837-845

25. Orton MR, Messiou C, Collins D, Morgan VA, Tessier J, Young H, et al. Diffusion-weighted MR imaging of metastatic abdominal and pelvic tumours is sensitive to early changes induced by a VEGF inhibitor using alternative diffusion attenuation models. Eur Radiol 2016;26:1412-1419

26. Winfield JM, deSouza NM, Priest AN, Wakefield JC, Hodgkin C, Freeman S, et al. Modelling DW-MRI data from primary and metastatic ovarian tumours. Eur Radiol 2015;25:2033-2040

27. Panagiotaki E, Walker-Samuel S, Siow B, Johnson SP, Rajkumar V, Pedley RB, et al. Noninvasive quantification of solid tumor microstructure using VERDICT MRI. Cancer Res 2014;74:19021912

28. Kim S, Jain M, Harris AB, Lee VS, Babb JS, Sigmund EE, et al. T1 hyperintense renal lesions: characterization with diffusionweighted MR imaging versus contrast-enhanced MR imaging. Radiology 2009;251:796-807

29. Taouli B, Thakur RK, Mannelli L, Babb JS, Kim S, Hecht EM, et al. Renal lesions: characterization with diffusion-weighted imaging versus contrast-enhanced MR imaging. Radiology 2009;251:398-407

30. Shen L, Zhou L, Liu X, Yang X. Comparison of biexponential and monoexponential DWI in evaluation of Fuhrman grading of clear cell renal cell carcinoma. Diagn Interv Radiol 2017;23:100-105

31. Chandarana H, Kang SK, Wong S, Rusinek H, Zhang JL, Arizono $S$, et al. Diffusion-weighted intravoxel incoherent motion imaging of renal tumors with histopathologic correlation. Invest Radiol 2012;47:688-696

32. Hectors SJ, Semaan S, Song C, Lewis S, Haines GK, Tewari $A$, et al. Advanced diffusion-weighted imaging modeling for 
prostate cancer characterization: correlation with quantitative histopathologic tumor tissue composition-a hypothesisgenerating study. Radiology 2018;286:918-928

33. Zhu L, Pan Z, Ma Q, Yang W, Shi H, Fu C, et al. Diffusion kurtosis imaging study of rectal adenocarcinoma associated with histopathologic prognostic factors: preliminary findings. Radiology 2017;284:66-76

34. Fujima N, Sakashita T, Homma A, Shimizu Y, Yoshida A, Harada $T$, et al. Advanced diffusion models in head and neck squamous cell carcinoma patients: goodness of fit, relationships among diffusion parameters and comparison with dynamic contrast-enhanced perfusion. Magn Reson Imaging 2017;36:16-23
35. Anderson SW, Barry B, Soto J, Ozonoff A, O'Brien M, Jara $H$. Characterizing non-gaussian, high b-value diffusion in liver fibrosis: stretched exponential and diffusional kurtosis modeling. J Magn Reson Imaging 2014;39:827-834

36. Zhong Y, Wang H, Shen Y, Guo A, Wang J, Kang S, et al. Diffusion-weighted imaging versus contrast-enhanced MR imaging for the differentiation of renal oncocytomas and chromophobe renal cell carcinomas. Eur Radiol 2017;27:49134922

37. Sadowski EA, Bennett LK, Chan MR, Wentland AL, Garrett AL, Garrett RW, et al. Nephrogenic systemic fibrosis: risk factors and incidence estimation. Radiology 2007;243:148-157 